\title{
Large capillary aneurysms secondary to retinal venous obstruction
}

\author{
JOEL SCHULMAN, LEE M. JAMPOL, AND MORTON F. GOLDBERG \\ From the University of Illinois Hospital Eye and Ear Infirmary, Chicago, Illinois, USA
}

SUMmARY Three patients had unusually large capillary aneurysms. Two of the patients had had previous branch vein occlusions, while a third had had a central retinal vein occlusion. The large capillary aneurysms were located in areas of ischaemic retina that were drained by the previously obstructed veins. Retinal ischaemia and, possibly, increased hydrostatic pressure following vein occlusion were postulated to result in the development of these atypical capillary aneurysms. They are similar in size to arterial macroaneurysms, but originate from the venous side of the capillary bed. Like typical capillary microaneurysms and arterial macroaneurysms, these large capillary aneurysms may result in visual loss from macular oedema, serous elevation of the macula, and circinate lipid exudation. Argon laser obliteration of the aneurysms appears to be effective therapy.

Retinal microaneurysms are capillary outpouchings that may be seen in association with diabetes mellitus, branch and central retinal vein occlusion, hyperviscosity syndromes, and other ophthalmological conditions. We have recently noted unusually large capillary aneurysms in 3 patients with previous retinal venous occlusions. These aneurysms originated from the capillary bed and were located at or near the terminal venous radicles. They superficially resembled arterial macroaneurysms. In 1 patient the aneurysms were associated with lipid exudation, serous detachment of the macula, and visual loss. Photocoagulation in this case was followed by visual improvement.

\section{Case reports}

CASE 1

A 57-year-old black woman had no ocular problems except presbyopia until 23 April 1979, when she noted blurred vision in her left eye. She was seen by an optometrist and was referred to the University of Illinois. Her medical history contained nothing relevant.

On examination, best corrected visual acuity was 20/20-1 OD and 20/200 OS. Applanation pressure was $20 \mathrm{mmHg}$ in each eye. Pupillary reactions were normal. Results of external and slit-lamp examinations were normal, except for early cortical catarac-

Correspondence to Lee M. Jampol, MD, 1855 West Taylor Street, Chicago, Illinois 60612, USA. tous changes. Ophthalmoscopic examination was unremarkable in the right eye. The left eye showed an old branch vein occlusion in the superotemporal region, with extensive retinal venous collaterals. Three large capillary aneurysms temporal to the macula were each surrounded by a partial lipid ring (Figs. 1 and 2). There was a serous elevation of the macula with marked lipid deposition.

Fluorescein angiography showed an irregular capillary bed in the distribution of the superotemporal vein near the disc. The 3 large capillary aneurysms filled slowly and showed late staining of their walls (Figs. 3 and 4). A subretinal collection of fluid was present in the macula.

Results of a general physical examination were normal. Laboratory tests, including 2-hour postprandial blood glucose, complete blood count, blood chemistries, sickle cell preparation, serum lysozyme, antinuclear antibody, rheumatoid factor, and serum protein electrophoresis, were normal. The FTA-ABS was reactive. A chest roentgenogram was normal.

The patient required 3 argon laser photocoagulation sessions to close the 3 leaking aneurysms. One month after treatment vision in the left eye improved to 20/40. The serous fluid in the macula resolved completely, and the lipid exudation showed moderate resolution.

CASE 2

A 79-year-old black man who had a history of an old central retinal vein occlusion in his left eye was 


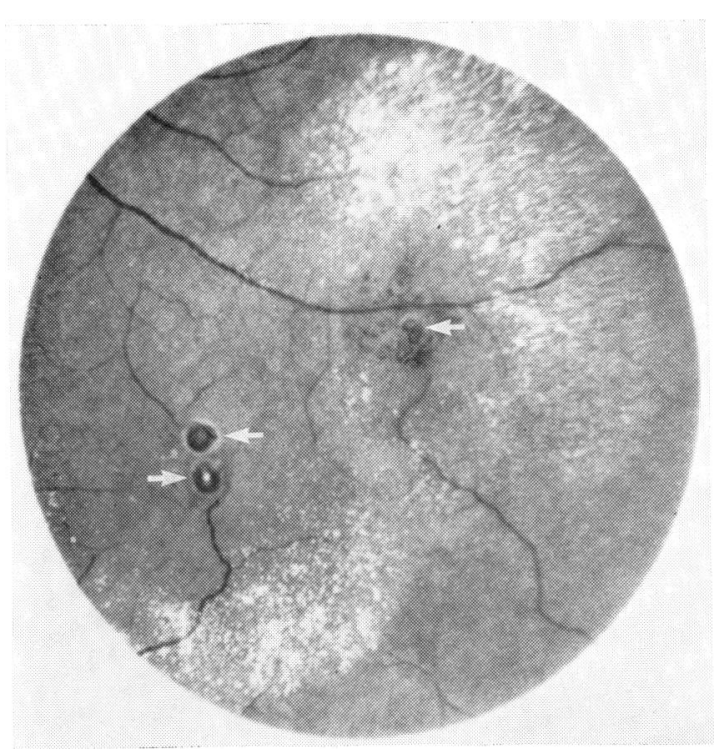

Fig. 1 Left eye, case 1. Three large capillary aneurysms temporal to the macula (arrows) are surrounded by circinate lipid exudate.

seen on 12 September 1979. His medical history contained nothing relevant.

Results of the ophthalmic examination revealed a corrected visual acuity of $20 / 25^{-2}$ OD and $20 / 400$ OS. Intraocular tensions by applanation tonometry were $17 \mathrm{mmHg}$ OD and $14 \mathrm{mmHg}$ OS. Pupillary responses and the external examination were normal. Slit-lamp examination showed vascular pannus on the medial aspect of the right cornea and inferior portion of the left cornea. Inferior lenticular cortical spoking was present but did not involve the visual axis. Examination of the right fundus was unremarkable. Visualisation of the left fundus was impaired by a resolving vitreous haemorrhage. The optic disc was slightly pale and showed disc neovascularisation. Areas of neovascularisation also were present in the temporal midperiphery and in the superonasal quadrant. The arteries and veins were narrowed. One large aneurysm was present below the superotemporal arcade near the papillomacular bundle (Fig. 5). Fluorescein angiography showed that the aneurysm was capillary in origin (Fig. 6).

Results of laboratory tests, including complete blood count, blood chemistries, serum lysozyme, FTA-ABS, sedimentation rate, glycosylated haemoglobin, 2-hour postprandial blood glucose, rheumatoid factor, antinuclear antibody, and serum protein electrophoresis were normal. A chest $x$-ray was normal. The patient's blood pressure was $146 / 80 \mathrm{mmHg}$.

\section{CASE 3}

A 67-year-old hypertensive white man was first seen in consultation on 26 September 1977 because of decreased vision in the left eye of at least 1 year's duration. He had been followed up by his local ophthalmologist, and vision had steadily improved without therapy. His medical history was unremarkable except for hypertension and gout.

Ocular examination revealed a corrected visual acuity of $20 / 20^{-1}$ OD and $20 / 25^{-2}$ OS. Intraocular tensions by applanation tonometry were $16 \mathrm{mmHg}$ OD and $14 \mathrm{mmHg}$ OS. Goldmann visual fields

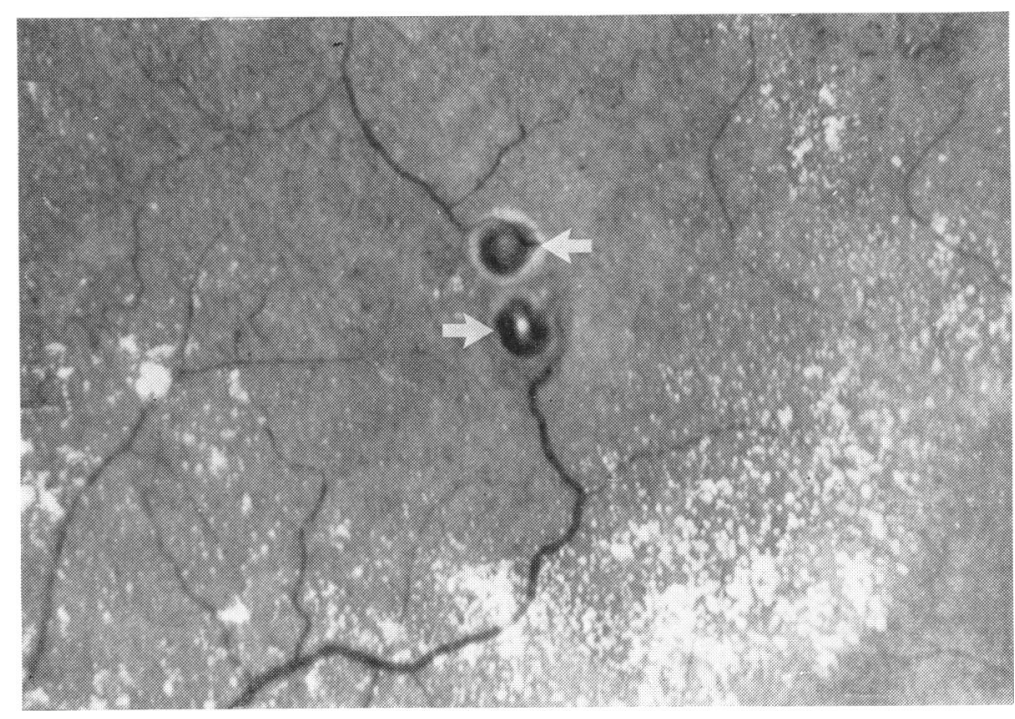

Fig. 2 Higher magnification of Fig. I shows the large capillary aneurysms (arrows). 
showed an absolute inferonasal scotoma in the left eye. Pupillary responses were normal. External and slit-lamp examinations were unremarkable. Results of ophthalmoscopy of the right eye were normal. In the left fundus a large patch of epipapillary neovascularisation extended into the temporal retina. In

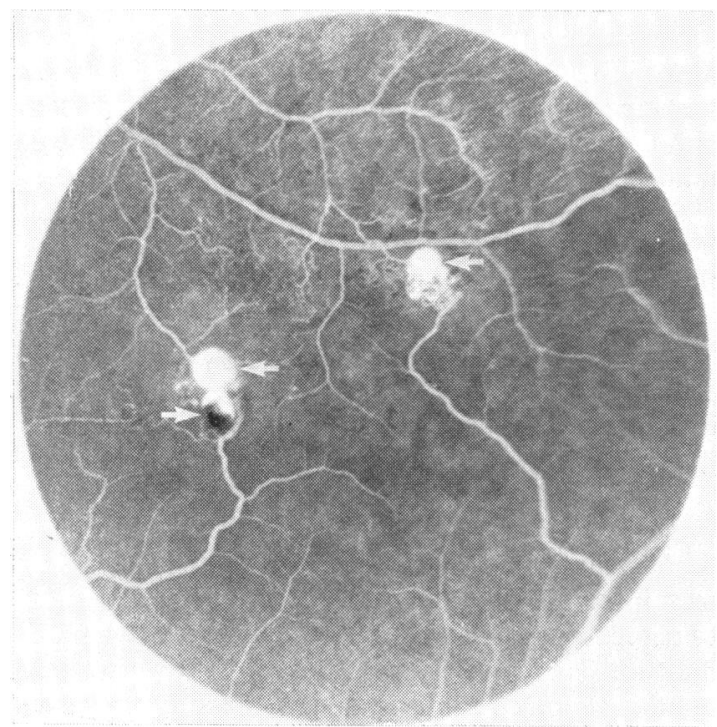

Fig. 3 Fluorescein angiogram of area seen in Fig. 1 shows filling of large capillary aneurysms (arrows). Note lowest aneurysm incompletely fills with fluorescein, possibly because its lumen is partially thrombosed.

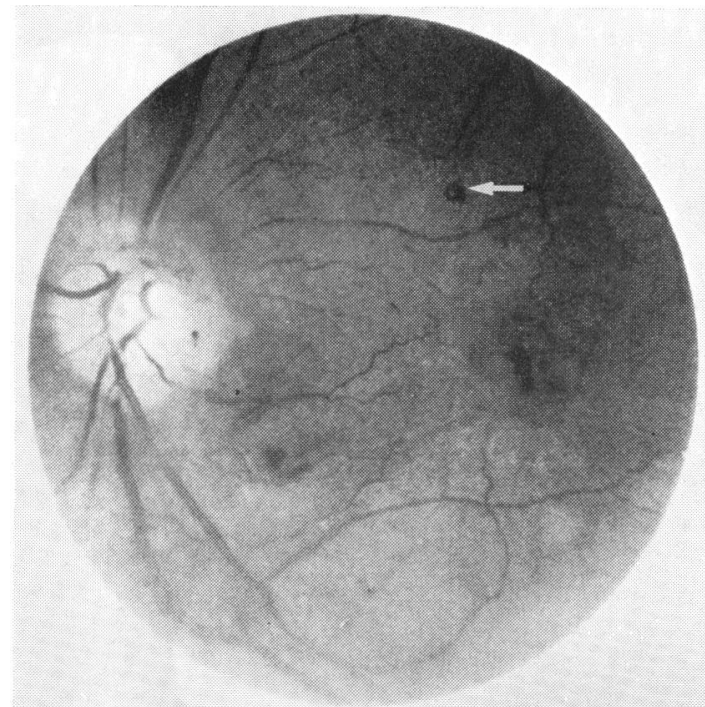

Fig. 5 Left eye, case 2. Large capillary aneurysm is seen (arrow). Disc neovascularisation is present. addition there was an old superotemporal branch vein occlusion with extensive retinal collaterals and microaneurysm formation. Two large capillary aneurysmal dilatations (Fig. 7) were located near the occluded vein. Fluorescein angiography revealed delayed filling of the superotemporal vein, late

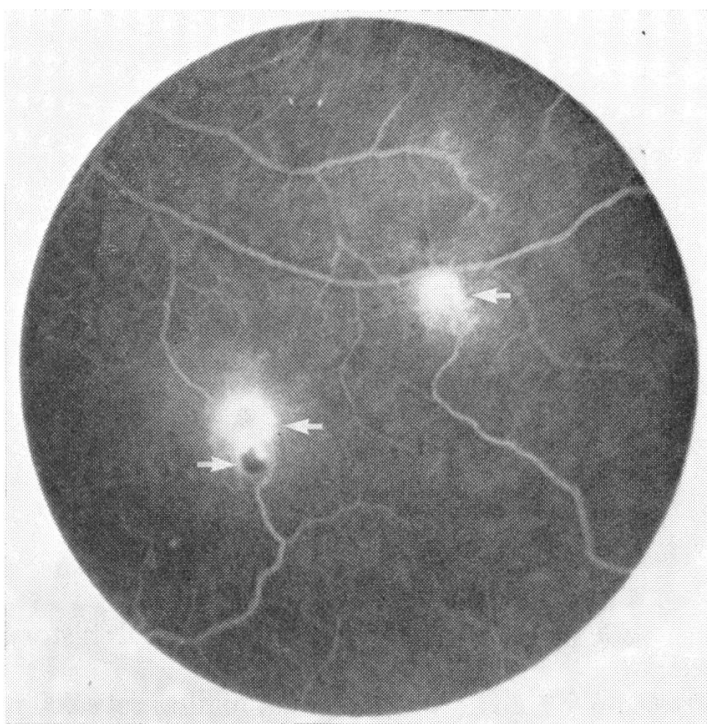

Fig. 4 Late view of left eye in case 1 reveals some leakage from aneurysms.

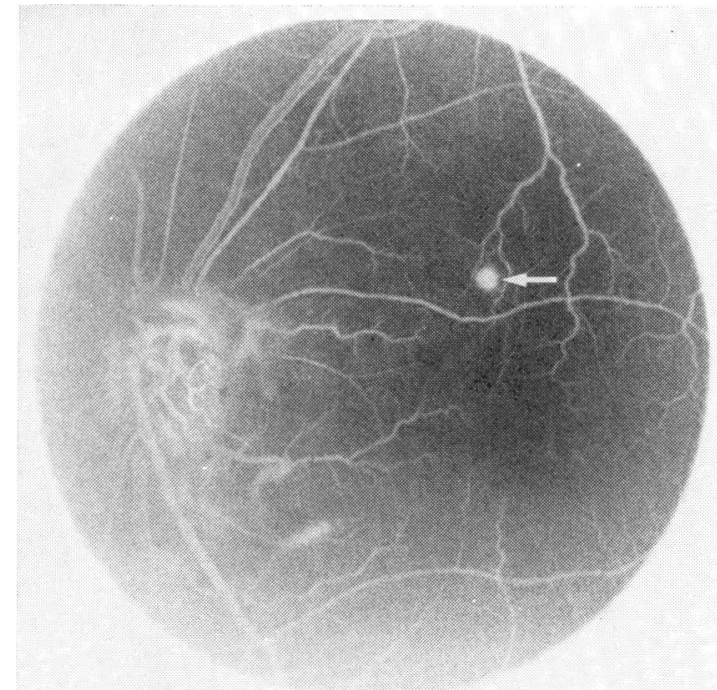

Fig. 6 Fluorescein angiogram of area seen in Fig. 5 shows filling of large capillary aneurysm (arrow) and leakage from disc neovascularisation. 


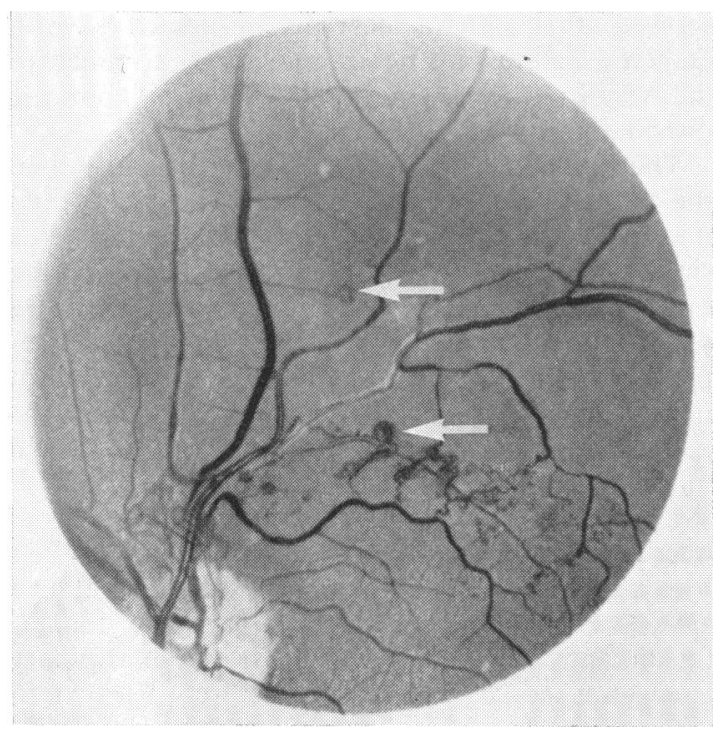

Fig. 7 Left eye, case 3. A large patch of epipapillary neovascularisation is seen. Old occlusion of the superotemporal vein is evident. Two large capillary aneurysms are present (arrows). Venous collaterals are seen.

staining of the walls of this vessel, and numerous collaterals and classic microaneurysms in the area of the vein occlusion. Fluorescein angiography showed that the 2 large aneurysms noted on fundus examination originated from capillaries (Fig. 8).

Results of laboratory testing, including complete blood count, blood chemistries, 2-hour postprandial blood glucose, rheumatoid factor, antinuclear antibody, FTA-ABS, glycosylated haemoglobin, and serum protein electrophoresis, were normal. His blood pressure was $180 / 120 \mathrm{mmHg}$.

Because of the disc neovascularisation the patient underwent argon laser treatment to the superotemporal quadrant of the left eye. On 17 October 1979 vision in the left eye was $20 / 25^{-2}$. Ophthalmoscopic examination of the left eye showed complete regression of the epipapillary neovascularisation and destruction of the 2 large capillary aneurysms. The patient was referred to his family physician for evaluation and treatment of uncontrolled hypertension.

\section{Discussion}

Large atypical capillary aneurysms were noted in 3 patients. All the patients were in good health with the exception of case 3 , who was hypertensive and had gout. A positive FTA-ABS was also noted in case 1 . None of the patients was diabetic. All had

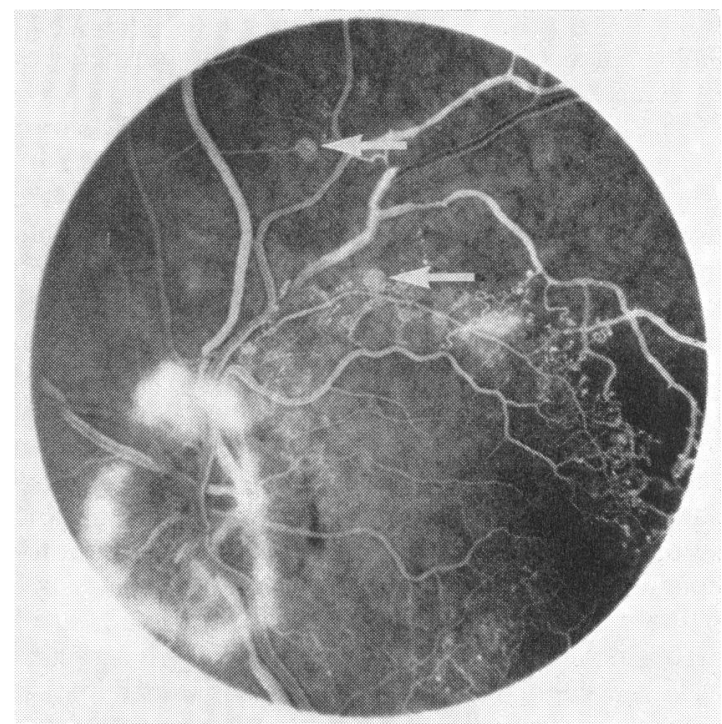

Fig. 8 Fluorescein angiogram of area seen in Fig. 7. The two large capillary aneurysms fill with fluorescein (arrows). Extensive capillary nonperfusion, classic microaneurysms, and leakage from the disc neovascularisation are visible.

unilateral eye disease with evidence of previous unilateral retinal venous obstruction. Two patients showed evidence of an old branch retinal vein occlusion, while the third had an old central retinal vein occlusion. Two of the patients experienced no visual symptoms related to their aneurysms. Case 1 had a localised serous detachment of the macula with circinate retinopathy. After argon laser obliteration of the aneurysms in this case the serous detachment resolved, there was a significant decrease in lipid exudation, and visual acuity improved.

The origin of aneurysms of the retinal vascular tree can be arterial, capillary, venous, or a combination of these. Isolated aneurysms on retinal arteries that arise within the first 3 orders of bifurcation have been termed macroaneurysms. ${ }^{1}$ Macroaneurysms are usually unilateral and tend to occur in elderly people with hypertension or arteriosclerosis. They may be postembolic. Macroaneurysms can cause retinal, preretinal, or vitreous haemorrhage, retinal oedema, or circinate retinopathy. Arterial aneurysms, presumably of a different pathogenesis, have also been reported in association with venous and capillary aneurysms in Coats's disease and Leber's miliary aneurysms (see below).

Small capillary aneurysms are called microaneurysms. Microaneurysms are found in a variety of conditions including vein occlusion, ${ }^{2}$ sickle cell anaemia,${ }^{3}$ chronic leukaemia, ${ }^{4}$ macroglobulinaemia, ${ }^{5}$ 
diabetes mellitus, hypertension, and glaucoma. ${ }^{6}$ They can also be present in apparently normal eyes of elderly patients.

The large capillary aneurysms described in our patients appear to be a variant of the microaneurysm. Measurements showed that these aneurysms are approximately the same size or slightly smaller than arterial macroaneurysms (approximately 100 to 250 $\mu \mathrm{m}$ in diameter). The aneurysms are significantly larger than microaneurysms (usually 30 to $90 \mu \mathrm{m}$ in diameter), ${ }^{7}$ but share many similar properties. Like microaneurysms or arterial macroaneurysms, large capillary aneurysms may be associated with a breakdown of the blood-retinal barrier and may cause circinate retinopathy, retinal oedema, and serous elevation of the macula (case 1). Photocoagulation can obliterate these leaking aneurysms, if the macula is threatened by oedema, exudate, or serous detachment.

Large capillary aneurysms in some patients fit into a spectrum of diseases that includes Leber's miliary aneurysms and Coats's disease. In 1908 Coats ${ }^{8}$ reported a relatively rare disorder in children distinguished by massive retinal exudation and vascular changes. An adult form of Coats's disease that involved the retinal periphery was later described. ${ }^{9}$ In 1912 Leber $^{10}$ reported on 13 young male patients with saccular dilatation of blood vessels and retinal degeneration. This disorder is now referred to as Leber's miliary aneurysms. Venous occlusion has been reported in both Coats's disease ${ }^{11}$ and Leber's miliary ansurysms. ${ }^{12}$

We have previously reported 1 case in which an arterial macroaneurysm subsequently progressed under observation to an adult Coats-like condition. ${ }^{1}$

In 1956 Reese $^{13}$ examined the possible relationship between Coats's disease and Leber's miliary aneurysms. He proposed that both entities are different expressions of the same disease process. $\mathrm{He}$ adopted the designation of retinal telangiectasia to describe changes of Leber's miliary aneurysms and considered Coats's disease to represent the more severe end of the same disease spectrum. Gass ${ }^{14}$ included in the definition of retinal telangiectasia focal and sometimes large capillary aneurysms somewhat similar to the isolated retinal lesions described in this report.

The location of large capillary aneurysms in our cases supports the concept that their development is similar to microaneurysm formation following vein occlusion. The aneurysms were situated on the venular side of the capillary network at or near the end of small venous branches. Okun and Collins ${ }^{15}$ showed that microaneurysms following experimental branch vein occlusion in dogs occur exclusively on the venular capillaries. In contrast Cogan et al. ${ }^{16}$ showed that microaneurysms in patients with diabetes mellitus are not preferentially confined to the venous side of the capillary plexus but are distributed throughout the capillary system.

The pathophysiological mechanisms involved in the formation of large capillary aneurysms and microaneurysms following vein occlusion may be similar. Wise ${ }^{7}$ postulated that microaneurysms are incomplete attempts at neovascularisation. $\mathrm{He}$ suggested that the ischaemic retina releases a vasoproliferative factor that stimulates the formation of new blood vessels; this process is aborted when the tissue's need for oxygen is met, thereby resulting in microaneurysm formation. The failure to demonstrate the development of neovascularisation from microaneurysms is strong evidence against Wise's hypothesis. However, microaneurysms are almost always associated with areas of capillary nonperfusion and retinal ischaemia. The large capillary aneurysms noted in our patients were located in areas of poorly perfused retina that were drained by previously obstructed veins. Thus ischaemia may have played a role in their origin. In addition a simple transient or prolonged increase in hydrostatic venous pressure affecting a localised, diseased portion of a capillary could have contributed to the pathogenesis of these large capillary aneurysms. ${ }^{17}$

Supported in part by grant EY 02214 and EY 1792 from the National Eye Insticute; grant HL 15168 from the National Heart, Lung, and Blood Institute, Bethesda, Maryland; and by an unrestricted grant from the Illinois Society for the Prevention of Blindness.

Norbert Jednock and Tom Young assisted in the preparation of the photographs.

\section{References}

1 Asdourian GK, Goldberg MF, Jampol LM, Rabb, M. Retinal macroaneurysms. Arch Ophthalmol 1977; 95: 624-8.

2 Wise GN. Macular changes after venous obstruction. Arch Ophthalmol 1957; 58: 544-57.

3 Stevens TS, Busse B, Lee CB, Woolf MB, Galinos SO, Goldberg MF. Sickling hemoglobinopathies: macular and perimacular vascular abnormalities. Arch Ophthalmol 1974; 92 : 455-63.

4 Jampol LM, Goldberg MF, Busse B. Peripheral retinal microaneurysms in chronic leukemia. Am J Ophthalmol 1975; 80: 242-48.

5 Ashton N. Studies of the retinal capillaries in relation to diabetic and other retinopathies. $\mathrm{Br} \mathrm{J}$ Ophthalmol 1963; 47: 521-38.

6 Ashton N. Retinal micro-aneurysms in the non-diabetic subjecr. Br J Ophthalmol 1951; 35: 189-212.

7 Wise GN. Retinal microaneurysms. Arch Ophthalmol 1957; 57: 151-6.

8 Coats G. Forms of retinal disease with massive exudation. $R$ Lond Ophthalmol Hosp Rep 1907-8; 17: 440-525.

9 Henkind P, Morgan G. Peripheral retinal angioma with exudative retinopathy in adults (Coats's lesion). $\mathrm{Br} J$ Ophthalmol 1966; 50: 2-11. 
10 Leber T. Uber eine durch Vorkommen multipler Miliaraneurysmen charakterisierte Form von Retinaldegeneration. Albrecht von Graefes Arch Klin Ophthalmol 1912; $81:$ 1-14.

11 Egerer I, Tasman W, Tomer TL. Coats' disease. Arch Ophthalmol 1974; 92: 109-12.

12 Wegener JK. Leber's retinal degeneration with miliary aneurysms. Acta Ophthalmol (Kbh) 1969; 47: 108-14.

13 Reese AB. Telangiectasis of the retina and Coats' disease. Am J Ophthalmol 1956; 42: 1-8.

14 Gass JDM. A fluorescein angiographic study of macular dysfunction secondary to retinal vascular disease: $\mathrm{V}$.
Retinal telangiectasis. Arch Ophthalmol 1968; 80: 592605.

15 Okun E, Collins EM. Histopathology of experimental photocoagulation in the dog eye: Part III. Microaneurysm-like formations following branch vein occlusion. Am J Ophthalmol 1963; 56: 40-5.

16 Cogan D, Toussaint D, Kuwabara T. Retinal vascular patterns: IV. Diabetic retinopathy. Arch Ophthalmol 1961; 66: 366-78.

17 Goldberg MF. Diseases affecting the inner blood-retinal barrier. In: Cunha-Vaz JG, ed. Blood-Retinal Barrier. New York: Plenum Press, 1980: 309-64. 